\title{
The Pressure Boundary Condition and the Pressure as Lagrangian for Water Waves
}

\section{Thomas J. Bridges ${ }^{1}$}

Received: 24 July 2018 / Accepted: 2 January 2019 / Published online: 11 March 2019

(C) The Author(s) 2019

\begin{abstract}
The pressure boundary condition for the full Euler equations with a free surface and general vorticity field is formulated in terms of a generalized Bernoulli equation deduced from the Gavrilyuk-Kalisch-Khorsand conservation law. The use of pressure as a Lagrangian density, as in Luke's variational principle, is reviewed and extension to a full vortical flow is attempted with limited success. However, a new variational principle for time-dependent water waves in terms of the stream function is found. The variational principle generates vortical boundary conditions but with a harmonic stream function. Other aspects of vorticity in variational principles are also discussed.
\end{abstract}

Keywords Oceanography · Lagrangian · Vorticity · Streamfunction · Variational principle

\section{Introduction}

The dynamic boundary condition in the theory of water waves reduces, in the case of inviscid flow, to continuity of the pressure field. In inviscid irrotational flow this condition is replaced by the Bernoulli equation evaluated at the surface. In this paper, it is shown that there is a generalized Bernoulli equation valid for all inviscid flows. Restricting to two space dimensions with a free surface at $y=h(x, t)$, the Bernoulli equation is

$$
\Lambda_{t}+U \Lambda_{x}+g h-\frac{1}{2} U^{2}-\frac{1}{2} V^{2}+P_{a}=f(t)
$$

where $(U, V)$ is the velocity field evaluated at the free surface, $P_{a}(x, t)$ is the imposed external pressure field, and $f(t)$ is an arbitrary function of time. The function $\Lambda(x, t)$

$凶$ Thomas J. Bridges

t.bridges@surrey.ac.uk

1 Department of Mathematics, University of Surrey, Guildford, Surrey GU2 7XH, England 
is a generalized potential function

$$
\Lambda_{x}=U+V h_{x}
$$

This potential is just an anti-derivative as it places no restriction on the velocity field. However, it reduces to a classical velocity potential when the flow is irrotational. The Bernoulli Eq. (1.1) is implicit in GAVRILUK ET AL. [8]. They introduce a conservation law at the free surface and the Bernoulli equation here (1.1) is obtained by integration using (1.2). Both (1.2) and (1.1) are exact without restriction on the velocity or vorticity fields.

The pressure can be used as a density for a Lagrangian variational principle for water waves and this strategy forms the basis of Luke's variational principle [13]. The use of pressure as a Lagrangian density is reviewed and then the Bernoulli Eq. (1.1) is extended into the fluid interior to form a Lagrangian. However, this variational principle does not produce all the required governing equations and boundary conditions.

By introducing a stream function, to automatically satisfy the continuity equation, the Bernoulli Eq. (1.1) leads to a new variational formulation for water waves, with vortical boundary conditions, but vanishing vorticity in the interior, via a harmonic stream function. An interesting outcome of this variational principle is that $\delta L / \delta h=0$ gives precisely the vortical Bernoulli Eq. (1.1) with $(U, V)$ replaced by $\left(\psi_{y},-\psi_{x}\right)$ evaluated at the free surface, and $\delta L /\left.\delta \psi\right|^{y=h}=0$ generates the identity (1.2).

An outline of the paper is as follows. The governing equations are reviewed in $\S 2$ and the Bernoulli Eq. (1.1) derived. In $\S 3$ the Bernoulli equation is extended into the interior and it is proposed as the basis of a variational principle for vortical free surface flow. The new variational principle with the stream function representation is proposed in $§ 5$. In the concluding remarks, other aspects of vorticity in variational principles are discussed.

\section{Euler Equations for Water Waves}

The Euler equations for free surface flow are recorded here for reference. The fluid is inviscid and incompressible, with constant density $\rho$ absorbed into the pressure. The fluid domain consists of the set of all $(x, y) \in \mathbb{R}^{2}$ such that $0<y<h(x, t)$ with $x \in \mathbb{R}$ and $t \geq 0$. The governing equations and boundary conditions for the velocity field $(u, v)$ and pressure field $p$ are

$$
\left.\begin{array}{l}
u_{t}+u u_{x}+v u_{y}+p_{x}=0 \\
v_{t}+u v_{x}+v v_{y}+p_{y}+g=0 \\
u_{x}+v_{y}=0
\end{array}\right\} 0<y<h(x, t), \quad x \in \mathbb{R}, t>0 .
$$

The boundary conditions are

$$
v=0 \text { at } y=0 \text {, }
$$




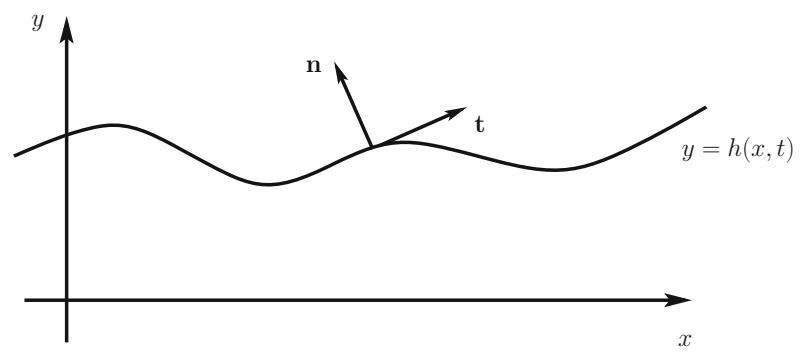

Fig. 1 Schematic of water wave problem

and at the free surface

$$
p=p_{a} \text { and } h_{t}+u h_{x}=v \quad \text { at } y=h(x, t),
$$

where $p_{a}(x, y, t)$ is the external atmospheric pressure field. No particular boundary conditions are applied at fixed $x$ locations as the main focus is on the pressure field. At each point on the surface there is an orthonormal moving frame which can be taken to be $(\mathbf{t}, \mathbf{n})$ with

$$
\mathbf{t}=\frac{1}{\ell}\left(\begin{array}{c}
1 \\
h_{x}
\end{array}\right) \text { and } \mathbf{n}=\frac{1}{\ell}\left(\begin{array}{c}
-h_{x} \\
1
\end{array}\right), \quad \text { with } \ell=\sqrt{1+h_{x}^{2}} .
$$

\subsection{The Pressure Boundary Condition}

It is clear from the statement of the problem that an arbitrary function of time can be added to the pressure field without affecting the velocity field. Hence an equivalent boundary condition for the pressure at the free surface is

$$
\nabla p \cdot \mathbf{t}=\nabla p_{a} \cdot \mathbf{t}, \quad \text { at } y=h(x, t)
$$

or with

$$
P(x, t)=p(x, h(x, t), t) \quad \text { and } \quad P_{a}(x, t)=p_{a}(x, h(x, t), t),
$$

the pressure boundary condition is

$$
\frac{\partial P}{\partial x}=\frac{\partial P_{a}}{\partial x}, \quad \text { at } y=h(x, t)
$$

This boundary condition is interesting as the Euler equations can be directly substituted. Substituting for $p_{x}$ and $p_{y}$ from (2.1)

$$
-\frac{\partial P_{a}}{\partial x}=-\left.\left[p_{x}+p_{y} h_{x}\right]\right|^{y=h}=\left.\frac{D u}{D t}\right|^{y=h}+\left.\frac{D v}{D t}\right|^{y=h} h_{x}+g h_{x} .
$$


Although apparently more complicated, this equation simplifies when expressed in terms of surface variables. Let

$$
U(x, t)=u(x, h(x, t), t) \text { and } V(x, t)=v(x, h(x, t), t) .
$$

Then

$U_{t}+U U_{x}=\left.\left[u_{t}+u_{y} h_{t}+u u_{x}+u u_{y} h_{x}\right]\right|^{y=h}=\left.\left[\frac{D u}{D t}+u_{y}\left(h_{t}+u h_{x}-v\right)\right]\right|^{y=h}$,

with a similar expression for $V_{t}+U V_{x}$. Hence, modulo the kinematic free surface condition, (2.5) simplifies to

$$
-\frac{\partial P_{a}}{\partial x}=U_{t}+U U_{x}+\left(V_{t}+U V_{x}\right) h_{x}+g h_{x},
$$

or upon rearranging

$$
-\frac{\partial P_{a}}{\partial x}=K_{t}+\left(U K+g h-\frac{1}{2} U^{2}-\frac{1}{2} V^{2}\right)_{x},
$$

with

$$
K:=U+V h_{x}
$$

or

$$
K_{t}+\left(U K+g h-\frac{1}{2} U^{2}-\frac{1}{2} V^{2}+P_{a}\right)_{x}=0 .
$$

Hence the pressure boundary condition can be expressed as a conservation law. In fact this is the conservation law of Gavrilyuk et al. [8], hereafter the GKK conservation law. It is an exact conservation law at the free surface with no restriction on the velocity or vorticity field. It is shown in [8] that this conservation law also extends to the case where the surface is represented parametrically and so can be multi-valued.

\subsection{A Vortical Bernoulli Equation at the Free Surface}

It is now a short step to integrate (2.7) using the generalized potential $\Lambda$ defined in (1.2). Substituting $\Lambda$ into (2.7) renders it integrable to

$$
\Lambda_{t}+U \Lambda_{x}+g h-\frac{1}{2} U^{2}-\frac{1}{2} V^{2}+P_{a}=0
$$

modulo an arbitrary function of time, which can be absorbed into $\Lambda$. The Eq. (2.8) replaces the pressure boundary condition (2.3). It is exact with no restriction on the vorticity field. Combining (2.8) with the kinematic free surface boundary condition 
gives the following general boundary conditions at the free surface for the Euler equations

$$
\left.\begin{array}{rl}
\Lambda_{t}+U \Lambda_{x} & =\frac{1}{2} U^{2}+\frac{1}{2} V^{2}-g h-P_{a} \\
h_{t}+U h_{x} & =V
\end{array}\right\} \text { at } y=h(x, t)
$$

When the velocity field is expressed in terms of a velocity potential the boundary condition (2.8) reduces exactly to the classic Bernoulli boundary condition for time dependent irrotational flow with a free surface. To see this, take $(u, v)=\left(\phi_{x}, \phi_{y}\right)$ then

$$
\Lambda_{x}=K=U+V h_{x}=\left.\left[\phi_{x}+\phi_{y} h_{x}\right]\right|^{y=h}=\Phi_{x}
$$

where $\Phi(x, t):=\phi(x, h(x, t), t)$. With $\Lambda=\Phi$ (modulo an arbitrary function of time), and using

$$
\Phi_{t}+U \Phi_{x}=\phi_{t}+\phi_{x}^{2}+\phi_{y}^{2}, \quad \text { at } y=h
$$

it follows that

$$
\Lambda_{t}+U \Lambda_{x}+g h-\frac{1}{2} U^{2}-\frac{1}{2} V^{2}+P_{a}=\phi_{t}+\frac{1}{2}\left(\phi_{x}^{2}+\phi_{y}^{2}\right)+g h+P_{a}, \quad \text { at } y=h,
$$

and the latter equation is the classic irrotational Bernoulli equation at a free surface. Hence (2.8) is a true generalization of the Bernoulli boundary condition to free surface flow with vorticity.

\section{A Vortical Bernoulli Equation in the Interior}

In [8] the GKK conservation law is extended into the interior. The extension of $K$ into the interior is

$$
K^{\chi}:=u(t, x, \chi)+v(t, x, \chi) Y_{x},
$$

where $Y(t, x, \chi)$ is a semi-Lagrangian change of coordinates from $y$ to $\chi$ (in [8] the symbol $\lambda$ is used instead of $\chi$, but here $\lambda$ is used as a Lagrange multiplier). At each $t$ this change of coordinates foliates the $(x, y)$ domain by contact lines indexed by $\chi \in[0,1]$ with $\chi=0$ the bottom and $\chi=1$ the free surface (see pages 1805-1806 in [8] for further detail). Along constant $\chi$ curves the following conservation law is obtained in [8]

$$
\frac{\partial K^{\chi}}{\partial t}+\frac{\partial}{\partial x}\left(u K^{\chi}+g Y-\frac{1}{2} u^{2}-\frac{1}{2} v^{2}+p\right)=0, \quad \chi \text { fixed. }
$$


Define the $\chi$-dependent potential

$$
\frac{\partial \Lambda^{\chi}}{\partial x}:=u+v Y_{x}
$$

Then the following Bernoulli equation is generated in the interior

$$
\left(\Lambda^{\chi}\right)_{t}+u\left(\Lambda^{\chi}\right)_{x}+g Y-\frac{1}{2} u^{2}-\frac{1}{2} v^{2}+p=f(t),
$$

where $f(t)$ is an arbitrary function of time. Solving for $p(x, \chi, t)$ generates an explicit expression for $p$. As in Luke's variational principle, can this expression for $p$ form the basis of a Lagrangian variational principle for free surface flow with vorticity?

\section{Pressure as Lagrangian Density}

The idea that the pressure can play the role of a Lagrangian density when constructing variational principles for inviscid fluid flow has been widely used. The idea appears to have been first proposed by Hargreaves [11], used by Bateman [2] (see page 164 in [2]), Seliger and Whitham [14] and Casetta and Pesce [3], and forms the basis of Luke's variational principle [13]. It is sometimes called the Bateman-Luke variational principle.

On the other hand, the conventional approach to constructing variational principles in mechanics is to start with a Lagrangian density consisting of the kinetic energy minus the potential energy. When that is not sufficient constraints can be added with Lagrange multipliers (e.g. Dutykh and Clamond [4] and references therein).

For the case of water waves with an irrotational velocity field, the pressure as Lagrangian is precisely equal to the kinetic minus potential—as long as the right constraints are added (LUKE [13] has a remark to this effect). To see this, start with the exact Bernoulli equation for irrotational flow integrated over the flow field

$$
\int_{x_{1}}^{x_{2}} \int_{0}^{h}\left[p+\phi_{t}+\frac{1}{2}\left(\phi_{x}^{2}+\phi_{y}^{2}\right)+g y-f(t)\right] \mathrm{d} y \mathrm{~d} x=0,
$$

where $f(t)$ is an arbitrary function of time. Absorb $f(t)$ into $\phi$ or $p$ and re-cast as

$$
\int_{0}^{h} p \mathrm{~d} y=-\int_{0}^{h}\left[\phi_{t}+\frac{1}{2}\left(\phi_{x}^{2}+\phi_{y}^{2}\right)+g y\right] \mathrm{d} y .
$$

The integral over $x$ is understood, as it will not play a central role. The expression (4.1) shows that the integral of the pressure over the flow field is exactly equal to the density in Luke's variational principle.

On the other hand with an elementary integral by parts argument, the right hand side can be reformulated in terms of the kinetic and potential energies. Define

$$
\mathrm{KE}:=\int_{0}^{h} \frac{1}{2}\left(\phi_{x}^{2}+\phi_{y}^{2}\right) \mathrm{d} y \text { and } \mathrm{PE}:=\int_{0}^{h} g y \mathrm{~d} y,
$$


and noting that

$$
\int_{0}^{h} \phi_{t} \mathrm{~d} y=\frac{\partial}{\partial t} \int_{0}^{h} \phi \mathrm{d} y-\Phi h_{t}
$$

the pressure expression (4.1) can be recast as

$$
\begin{aligned}
\int_{0}^{h} p \mathrm{~d} y & =-\int_{0}^{h}\left[\phi_{t}+\frac{1}{2}\left(\phi_{x}^{2}+\phi_{y}^{2}\right)+g y\right] \mathrm{d} y \\
& =\Phi h_{t}-\mathrm{KE}-\mathrm{PE}-\frac{\partial}{\partial t} \int_{0}^{h} \phi \mathrm{d} y \\
& =\mathrm{KE}-\mathrm{PE}+\Phi\left(h_{t}+V_{n}\right)-2 \mathrm{KE}-\Phi V_{n}-\frac{\partial}{\partial t} \int_{0}^{h} \phi \mathrm{d} y,
\end{aligned}
$$

where

$$
V_{n}:=\left.\left[\phi_{x} h_{x}-\phi_{y}\right]\right|^{y=h}
$$

But

$$
\begin{aligned}
2 \mathrm{KE}+\Phi V_{n} & =\int_{0}^{h}\left(\phi_{x}^{2}+\phi_{y}^{2}\right) \mathrm{d} y+\left.\Phi\left(\phi_{x} h_{x}-\phi_{y}\right)\right|^{y=h} \\
& =-\int_{0}^{h} \phi \Delta \phi \mathrm{d} y-\left.\phi \phi_{y}\right|_{y=0}+\frac{\partial}{\partial x} \int_{0}^{h} \phi \phi_{x} \mathrm{~d} y .
\end{aligned}
$$

Hence

$$
\int_{0}^{h} p \mathrm{~d} y=\mathrm{KE}-\mathrm{PE}+\left.\Phi\left(h_{t}+\phi_{x} h_{x}-\phi_{y}\right)\right|^{y=h}+\partial B,
$$

where

$$
\partial B=\int_{0}^{h} \phi \Delta \phi \mathrm{d} y+\left.\phi \phi_{y}\right|_{y=0}-\frac{\partial}{\partial x} \int_{0}^{h} \phi \phi_{x} \mathrm{~d} y-\frac{\partial}{\partial t} \int_{0}^{h} \phi \mathrm{d} y .
$$

The integral of the pressure is equal to the conventional kinetic energy minus potential energy Lagrangian density if the kinematic free surface boundary condition is applied as a constraint, taking into account that $\phi$ is harmonic and $\phi_{y}$ vanishes at $y=0$ with appropriate conditions at $x$ boundaries (e.g. Neumann boundary conditions or periodic boundary conditions at $x=x_{1}$ and $x=x_{2}$ ), and variations with fixed endpoint conditions (e.g. $\left.\delta \phi\right|_{t_{1}} ^{t_{2}}=0$ ).

\subsection{Pressure as Lagrangian Density—With Vorticity}

The above argument, and the vast literature on "pressure as Lagrangian density", suggests that the pressure field for free surface flow with vorticity generated by the Bernoulli Eq. (3.4) can serve as a Lagrangian density 


$$
\delta \int_{t_{1}}^{t_{2}} \int_{x_{1}}^{x_{2}} \int_{0}^{1}\left[\left(\Lambda^{\chi}\right)_{t}+u\left(\Lambda^{\chi}\right)_{x}+g Y-\frac{1}{2} u^{2}-\frac{1}{2} v^{2}\right] Y_{\chi} \mathrm{d} \chi \mathrm{d} x \mathrm{~d} t=0 .
$$

As the reader can easily verify, this variational principle fails to produce the governing equations and boundary conditions for the full Euler equations with a free surface. It does generate the dynamic free surface boundary condition (assuming $\delta Y_{\chi} / \delta h \neq 0$ ) but does not capture the interior equations or kinematic condition. The first obvious problem is that $\Lambda^{\chi}$ is not defined and so (3.3) should be added as a constraint

$$
\begin{aligned}
& \delta \int_{t_{1}}^{t_{2}} \int_{x_{1}}^{x_{2}} \int_{0}^{1}\left[\left(\Lambda^{\chi}\right)_{t}+u\left(\Lambda^{\chi}\right)_{x}+g Y-\frac{1}{2} u^{2}-\frac{1}{2} v^{2}+\lambda\left(u+v Y_{x}-\left(\Lambda^{\chi}\right)_{x}\right)\right] \\
& Y_{\chi} \mathrm{d} \chi \mathrm{d} x \mathrm{~d} t=0,
\end{aligned}
$$

where $\lambda$ is a Lagrange multiplier. However, this variational principle is also ineffective. It fails to produce evolution equations in the interior. One can continue to add constraints but there is no obvious strategy for generating all the governing equations and boundary conditions.

It appears that the idea of using the pressure as a Lagrangian density is effective only in the case of irrotational flows. In this case, the kinetic energy generates the interior equation (harmonic velocity potential or harmonic stream function). Any more complex interior equations, like the Euler equations, do not appear to be captured by a pressure-based variational principle.

\section{Stream-Function Based Variational Formulation}

Represent the velocity in terms of a stream function,

$$
u=\psi_{y}, \quad v=-\psi_{x}, \quad \text { with } \Psi(x, t):=\psi(x, h(x, t), t) .
$$

The stream function satisfies the continuity equation exactly and there is no assumption of irrotationality at this point.

Now go back to (4.3) and instead of using pressure as Lagrangian density, start with a variational principle based on kinetic minus potential energy, and then add constraints as appropriate.

A posteriori it will become apparent that the kinematic free surface boundary condition will have to be added as a constraint. In terms of the stream function it takes the form

$$
0=h_{t}+u h_{x}-v=h_{t}+\psi_{x}+\psi_{y} h_{x}=h_{t}+\Psi_{x} \quad \text { at } y=h(x, t),
$$

where $\Psi$ is defined in (5.1). The constraint $\psi_{x}=0$ at $y=0$ is also included for a tidy representation of the bottom boundary condition. 
The proposed variational principle is

$$
\delta \int_{t_{1}}^{t_{2}} \int_{x_{1}}^{x_{2}} L \mathrm{~d} x \mathrm{~d} t=0
$$

with

$$
L=\int_{0}^{h} \frac{1}{2}\left(\psi_{x}^{2}+\psi_{y}^{2}\right) \mathrm{d} y-\frac{1}{2} g h^{2}+\left.\Lambda\left(h_{t}+\Psi_{x}\right)\right|^{y=h}+\left.\lambda \psi_{x}\right|_{y=0},
$$

where $\Lambda(x, t)$ and $\lambda(x, t)$ are Lagrange multipliers. The symbol $\Lambda$ anticipates a connection with (1.2).

Taking variations

$$
\begin{aligned}
\delta L= & \int_{0}^{h}\left(\psi_{x} \delta \psi_{x}+\psi_{y} \delta \psi_{y}\right) \mathrm{d} y+\left.\left[\frac{1}{2}\left(\psi_{x}^{2}+\psi_{y}^{2}\right)-g h\right]\right|^{y=h} \delta h \\
& +\left.\delta \Lambda\left(h_{t}+\Psi_{x}\right)\right|^{y=h}+\left.\Lambda\left(\delta h_{t}+\delta \Psi_{x}\right)\right|^{y=h} \\
& +\left.\delta \lambda \psi_{x}\right|_{y=0}+\left.\lambda \delta \psi_{x}\right|_{y=0} .
\end{aligned}
$$

But

$$
\begin{aligned}
& \int_{0}^{h}\left(\psi_{x} \delta \psi_{x}+\psi_{y} \delta \psi_{y}\right) \mathrm{d} y=\left.K \delta \psi\right|^{y=h}-\left.\psi_{y} \delta \psi\right|_{y=0}-\int_{0}^{h} \Delta \psi \delta \psi \mathrm{d} y \\
& +\frac{\partial}{\partial x} \int_{0}^{h} \psi_{x} \delta \psi \mathrm{d} y
\end{aligned}
$$

using $K=\left.\left[u+v h_{x}\right]\right|^{y=h}=\left.\left[\psi_{y}-\psi_{x} h_{x}\right]\right|^{y=h}$, and

$$
\begin{aligned}
\Lambda\left(\delta h_{t}+\delta \Psi_{x}\right) & =-\Lambda_{t} \delta h-\Lambda_{x} \delta \Psi+(\Lambda \delta h)_{t}+(\Lambda \delta \Psi)_{x} \\
& =-\Lambda_{t} \delta h-\left.\Lambda_{x} \delta \psi\right|^{y=h}-\Lambda_{x} \psi \psi_{y} \delta h+(\Lambda \delta h)_{t}+(\Lambda \delta \Psi)_{x} \\
& =-\left(\Lambda_{t}+U \Lambda_{x}\right) \delta h-\left.\Lambda_{x} \delta \psi\right|^{y=h}+(\Lambda \delta h)_{t}+(\Lambda \delta \Psi)_{x}
\end{aligned}
$$

using $U=\left.\psi_{y}\right|^{y=h}$ and $\delta \Psi=\left.\delta \psi\right|^{y=h}+\left.\psi_{y}\right|^{y=h} \delta h$. Substituting into $\delta L$ in (5.4),

$$
\begin{aligned}
\delta L= & \left.\left(K-\Lambda_{x}\right) \delta \psi\right|^{y=h}+\left.\delta \Lambda\left(h_{t}+\Psi_{x}\right)\right|^{y=h} \\
& -\left.\left[\Lambda_{t}+U \Lambda_{x}-\frac{1}{2}\left(\psi_{x}^{2}+\psi_{y}^{2}\right)+g h\right]\right|^{y=h} \delta h \\
& -\int_{0}^{h} \Delta \psi \delta \psi \mathrm{d} y+\left.\delta \lambda \psi_{x}\right|_{y=0}+\left.\left(\psi_{y}-\lambda_{x}\right) \delta \psi\right|_{y=0}+\partial B .
\end{aligned}
$$

where

$$
\partial B=\frac{\partial}{\partial x} \int_{0}^{h} \psi_{x} \delta \psi \mathrm{d} y+\left.(\lambda \delta \psi)_{x}\right|_{y=0}+(\Lambda \delta h)_{t}+(\Lambda \delta \Psi)_{x} .
$$


By assuming fixed endpoint conditions or appropriate boundary conditions the terms in $\partial B$ vanish. Then setting each term in $\delta L$ to zero gives the governing equations and boundary conditions: at the free surface

$$
h_{t}+\Psi_{x}=0 \text { and } \Lambda_{t}+U \Lambda_{x}+g h-\frac{1}{2}|\nabla \psi|^{2}=0 \text { at } y=h \text {, }
$$

in the interior

$$
\psi_{x x}+\psi_{y y}=0 \text { for } 0<y<h
$$

and at the bottom

$$
\psi_{x}=0 \text { at } y=0
$$

with the constraints

$$
\Lambda_{x}=\left.\left[\psi_{y}-\psi_{x} h_{x}\right]\right|^{y=h} \text { and } \lambda_{x}=\left.\psi_{y}\right|_{y=0}
$$

There are several interesting outcomes emerging from this variational principle. The dynamic free surface boundary condition arises naturally as the vortical Bernoulli Eq. (2.8),

$$
\frac{\delta L}{\delta h}=-\left.\left[\Lambda_{t}+U \Lambda_{x}-\frac{1}{2}\left(\psi_{x}^{2}+\psi_{y}^{2}\right)+g h\right]\right|^{y=h},
$$

and $\Lambda$ generates the generalized potential (1.2)

$$
\left.\frac{\delta L}{\delta \psi}\right|^{y=h}=K-\Lambda_{x} .
$$

In terms of the stream function this latter equation is

$$
\Lambda_{x}=\ell \nabla \psi \cdot \mathbf{n} \text { at } y=h,
$$

where $\mathbf{n}$ is the outward pointing normal at the free surface (2.4). Hence the stream function gradient at the surface has the decomposition

$$
\ell \nabla \psi=-h_{t} \mathbf{t}+\Lambda_{x} \mathbf{n} \text { at } y=h(x, t) .
$$

On the other hand, a curiosity of this variational principle is that it does not impose irrotationality a priori, yet the outcome is zero vorticity. The free surface boundary conditions (5.7) and the bottom boundary condition are exactly the same when the flow has vorticity. The only thing missing is the vorticity $\omega$ on the right hand side of (5.8). The variational principle is unaware of this vorticity field. 


\subsection{Natural Versus Imposed Boundary Conditions}

Variational principles based on a velocity potential, as in Luke's variational principle generate natural boundary conditions at rigid boundaries. This can be clearly seen with the boundary condition at $y=0$ generated by Luke's variational principle. Take the variation of the kinetic energy term and isolate the term at $y=0$,

$$
\delta \int_{0}^{h} \frac{1}{2}\left(\phi_{x}^{2}+\phi_{y}^{2}\right) \mathrm{d} y=-\left.\phi_{y} \delta \phi\right|_{y=0}+\cdots .
$$

Taking $\left.\delta \phi\right|_{y=0}$ to be arbitrary then generates the correct boundary condition $\left.\phi_{y}\right|_{y=0}=$ 0 . It is a natural boundary condition.

On the other hand, consider the case where the stream function is used in the kinetic energy and the same argument is applied

$$
\delta \int_{0}^{h} \frac{1}{2}\left(\psi_{x}^{2}+\psi_{y}^{2}\right) \mathrm{d} y=-\left.\psi_{y} \delta \psi\right|_{y=0}+\cdots .
$$

Now the natural boundary condition generated by the varational principle, $\left.\psi_{y}\right|_{y=0}=0$, is false. Therefore, either constrained variations are required ( $\psi$ is a constant on $y=0$ so $\left.\delta \psi\right|_{y=0}$ ), or the correct boundary conditions can be imposed as a constraint. It is the latter approach that is the strategy here and it is very effective. It is implemented via the term $\left.\lambda \psi_{x}\right|_{y=0}$ in (5.3). When boundary conditions are included at $x=x_{1}$ and $x=x_{2}$ a similar strategy is required to ensure vanishing of the terms in $\partial B$ in (5.6).

\section{Concluding Remarks}

Integrating the GKK conservation law gives a Bernoulli equation and a representation of the pressure boundary condition at the free surface in terms of this Bernoulli equation. However, generating the full equations with vorticity via a Lagrangian variational principle has had mixed success.

Although the interest in this paper has been restricted to Lagrangian variational principles, it is noteworthy that on the Hamiltonian side non-canonical Lie-Poisson brackets can be introduced to represent vorticity (e.g. Lewis et al. [12]), and when the vorticity is constant canonical Hamiltonian formulations can be constructed (e.g. Wahlén [15] and Groves and Wahlén [10]).

On the other hand, vorticity can be generated in a Lagrangian variational principle by introducing a Clebsch representation for the velocity field. It was already noted by Bateman [2] (see \$2.52 of [2]) that a Clebsch representation for the velocity field

$$
u=\phi_{x}+\alpha \beta_{x} \text { and } v=\phi_{y}+\alpha \beta_{y},
$$

can be used to capture the vorticity field,

$$
\omega=\alpha_{x} \beta_{y}-\alpha_{y} \beta_{x}
$$


Moreover a Bernoulli equation can be generated as well as a Lagrangian variational principle. Luke notes this as well (see Eq. (12) in [13]). However, Clebsch variables are difficult to work with: they are not unique, can have difficulty with boundary conditions (e.g. Fukagawa and Fujitani [6]), and can have singularities in the potentials even when the velocity field is smooth (e.g. Graham and Henyey [9]).

Another approach, advocated in Gavrilyuk [7], is to use virtual motions and virtual displacements in the Lagrangian setting mapped to the Eulerian setting. A group theoretic approach for the Lagrange to Euler map is Euler-Poincaré reduction resulting in constrained variations. Starting with a variational principle for the Lagrangian particle path description for water waves, where the vorticity arises naturally in the variational principle, and then performing reduction via the particle relabelling group, suggests that the velocity variations are not free and should be modified to

$$
\delta \mathbf{u}=\mathbf{w}_{t}+[\mathbf{u}, \mathbf{w}],
$$

where $\mathbf{w}$ is a free variation and $[\cdot, \cdot]$ is a Lie bracket of vector fields. The EulerPoincaré strategy is used in Cotter and Bokhove [5] and Alemi Ardakani et al. [1]. But constrained variations make analysis of the variational principle more difficult.

Instead of constrained variations, one can keep adding constraints until all effects are included (e.g. [4]). For example, the Lagrangian density

$$
L=\int_{0}^{h}\left[\frac{1}{2}\left(u^{2}+v^{2}\right)-g y+\lambda\left(u_{x}+v_{y}\right)+\mu\left(\omega-v_{x}+u_{y}\right)+\gamma \frac{D \omega}{D t}\right] \mathrm{d} y
$$

is of the form kinetic minus potential and enforces incompressibility and introduces vorticity. Taking $\gamma=0$, variations with respect to $u$ and $v$ generate the Hodge decomposition of the velocity field

$$
u=\lambda_{x}+\mu_{y} \text { and } v=\lambda_{y}-\mu_{x} .
$$

However including $\gamma \neq 0$ generates a more complicated velocity representation. Moreover, an additional constraint is required to capture the kinematic free surface boundary condition. On the other hand, with enough constraints, one may be able to recover the full Euler equations with a free surface and general vorticity field.

Open Access This article is distributed under the terms of the Creative Commons Attribution 4.0 International License (http://creativecommons.org/licenses/by/4.0/), which permits unrestricted use, distribution, and reproduction in any medium, provided you give appropriate credit to the original author(s) and the source, provide a link to the Creative Commons license, and indicate if changes were made.

\section{References}

1. Ardakani, H. Alemi, Bridges, T.J., Gay-Balmaz, F.,Huang, Y., Tronci, C.: (2018) A variational principle for fluid sloshing with vorticity, dynamically coupled to vessel motion, arXiv:1809.10909

2. Bateman, H.: Partial Differential Equations. Cambridge University Press, Cambridge (1964) 
3. Casetta, L., Pesce, C.P.: On Seliger and Whitham's variational principle for hydrodynamic systems from the point of view of "fictitious particles'. Acta Mech. 219, 181-184 (2011)

4. Clamond, D., Dutykh, D.: Practical use of variational principles for modeling water waves. Phys. D 241, 25-36 (2012)

5. Cotter, C., Bokhove, O.: Variational water-wave model with accurate dispersion and vertical vorticity. J. Eng. Math. 67, 33-54 (2010)

6. Fukagawa, H., Fujitani, Y.: Clebsch potentials in the variational principle for a perfect fluid. Prog. Theor. Phys. 124, 517-531 (2010)

7. Gavrilyuk, S.: Multiphase flow modeling via Hamilton's Principle. In: dell'Isola, F., Gavrilyuk, S. (eds.) Variational Models and Methods in Solid and Fluid Mechanics. CISM Courses and Lectures, vol. 535. Springer, Vienna (2011)

8. Gavrilyuk, S., Kalisch, H., Khorsand, Z.: A kinematic conservation law in free surface flow. Nonlinearity 28, 1805-1821 (2015)

9. Graham, C.R., Henyey, F.S.: Clebsch representation near points where the vorticity vanishes. Phys. Fluids 12, 744 (2000)

10. Groves, M.D., Wahlén, E.: Existence and conditional energetic stability of solitary gravity-capillary water waves with constant vorticity. Proc. Roy. Soc. Edin. A 145, 791-883 (2015)

11. Hargreaves, R.: A pressure integral as kinetic potential. Phil. Mag. 16, 436-444 (1908)

12. Lewis, D., Marsden, J., Montgomery, R., Ratiu, T.: The Hamiltonian structure for dynamic free boundary problems. Phys. D 18, 391-404 (1989)

13. Luke, J.C.: A variational principle for a fluid with a free surface. J. Fluid Mech. 27, 395-397 (1967)

14. Seliger, R.E., Whitham, G.B.: Variational principles in continuum mechanics. Proc. Roy. Soc. Lond. A 305, 1-25 (1968)

15. Wahlén, E.: A Hamiltonian formulation of water waves with constant vorticity. Lett. Math. Phys. 79, 303-315 (2007) 